\title{
Designing and Delivering a Tracheostomy Educational Webinar Program
}

Asit Arora ${ }^{1}$, Chloe Swords ${ }^{2}$, Lina Bergman ${ }^{3}$, Rachel Wilson-Jeffers ${ }^{4}$, Diane Randall ${ }^{5}$, Linda Morris ${ }^{6}$, Michael Brenner ${ }^{7}$

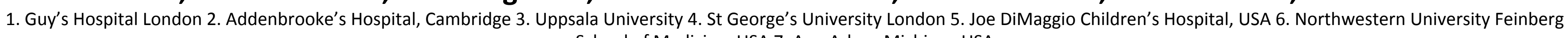

\section{Introduction}

School of Medicine, USA 7. Ann Arbor, Michigan USA

Caring for patients with tracheostomies involves the collaboration of multiple medical teams and caregivers. Regarding tracheostomies, the 2014 National Confidential Enquiry into Patient Outcome and Death report illustrated a complication rate of 25\% in a study over 2,500 tracheostomy patients ${ }^{1}$. Complications in this cohort may be life-threatening. The Global Tracheostomy Collaborative (GTC) is a quality improvement organisation which collaborates with healthcare professionals and patients to improve patient safety world-wide. The GTC emphasises the importance of education.

\section{Aims}

This project aimed to facilitate discussions and education around tracheostomy care within the global interprofessional medical community.

\section{Methods}

Global experts, including otolaryngologists, specialist nurses, speech therapists, caregivers and patients, led a series of live, interactive, international webinars covering various aspects of tracheostomy care (Figure 1-2). To assess and improve the educational benefit of the webinars, participants completed a survey before and after attending webinars. Responses were compared using the Mann Whitney $U$ test.

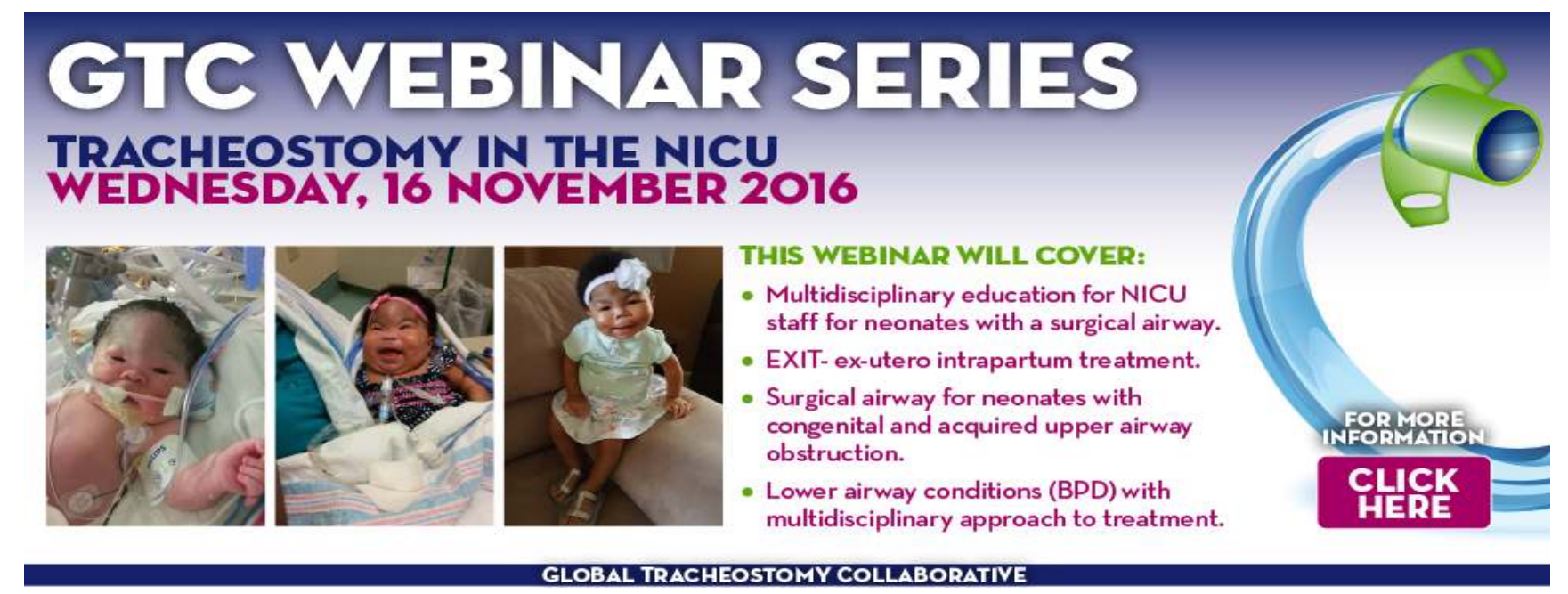

Figure 1. Global Tracheostomy Collaborative Webinar Series

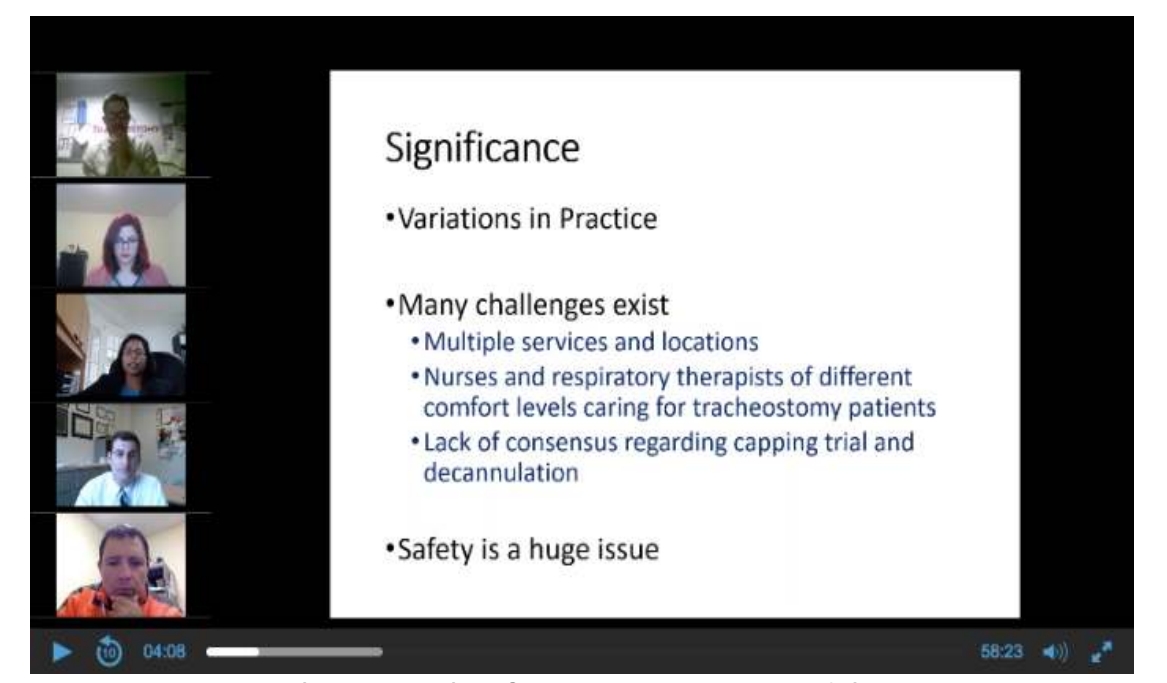

Figure 2. Webinar platform, as viewed by a participant

\section{Results}

- Participants completed 209 pre-evaluation surveys, and 107 post-evaluation surveys.

- A diverse range of professions were represented: speechlanguage pathology, physiotherapy, nursing and physicians.

- $86 \%(n=92)$ of participants would recommend attending the series to colleagues, and $83 \%(n=89)$ intended to integrate the learned concepts into their clinical practice.

- Participants indicated a significant gain in confidence in knowledge and ability to manage tracheostomy care encountered on a daily basis on a five-point Likert scale $(p<0.05)$ (Figure 3).

- Participants expressed practical ways of integrating concepts learned from the webinars into their workplaces, and intention to create standardised protocols involving multidisciplinary input and patient collaboration.

- Challenges relating to time-zone and the audibility of patient tutors with speaking valves were encountered.

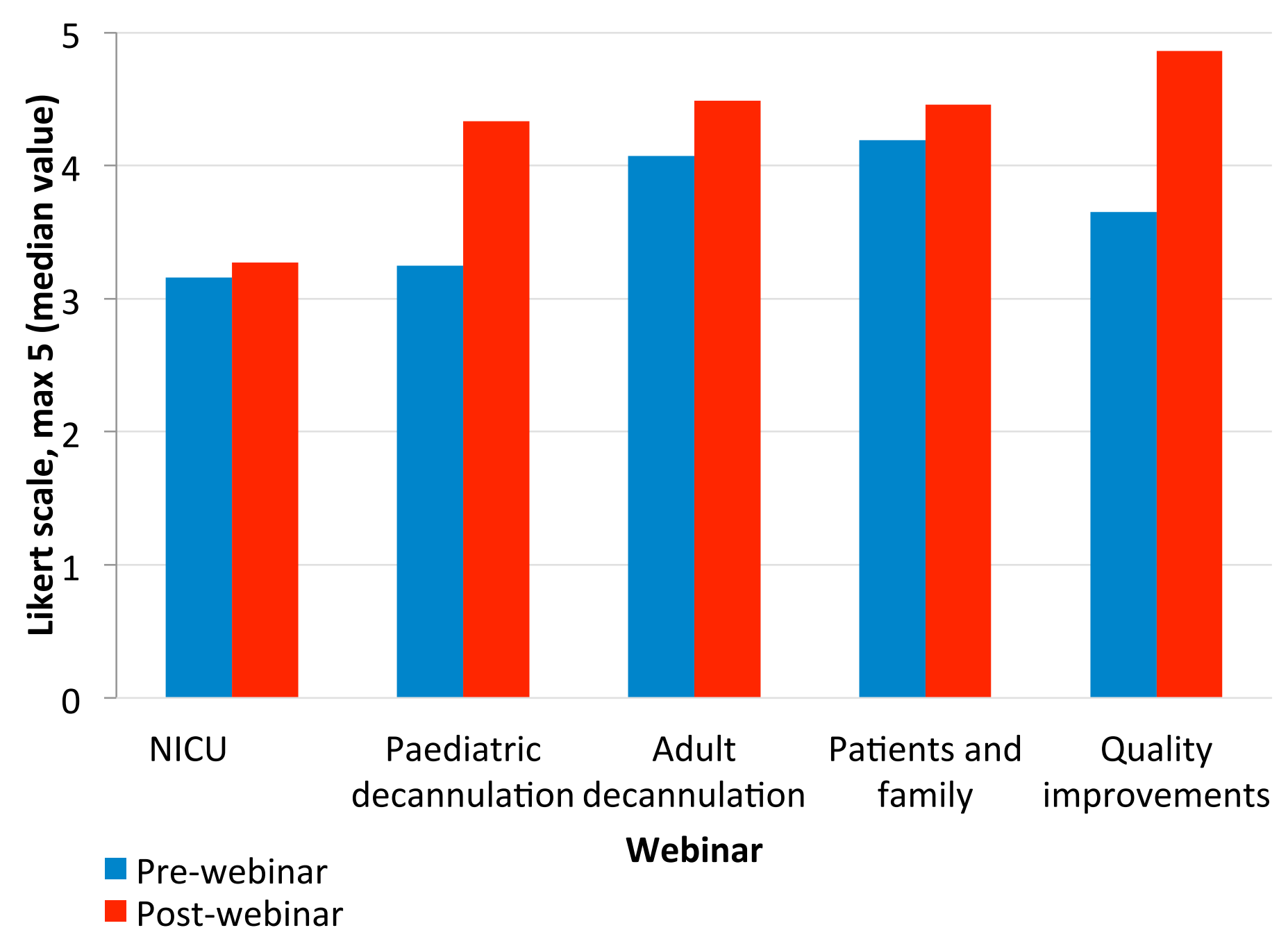

\section{Conclusion}

Figure 3. The effects of an educational webinar on the confidence in management in crucial aspects of tracheostomy care.

New technologies have allowed the global community to share from the experiences of experts. A unique aspect of this global webinar series is the ability for caregivers and medical professionals to ask questions to and learn from experts and clinician leaders in different countries. The benefits of online series are likely best realised when integrated with practical workshops.

\section{Key Points}

1. Tracheostomy care requires collaboration of multiple healthcare professionals, patients and carers.

2. Interactive webinars led by global experts are an effective and valuable method of educating healthcare professionals. 